\title{
Enrichir le savoir être des résidents en médecine par des stratégies pédagogiques diversifiées influençant leurs priorités
}

\section{Enhancing the life skills of medical residents through diversified educational strategies that have an impact on their priorities}

\author{
Bruno FORTIN ${ }^{1}$ \\ 1 Unité de Médecine Familiale Charles-Lemoyne, 299 Bd. Sir-Wilfrid-Laurier, suite 201, J4R 2L1 Saint-Lambert (Québec), \\ Canada
}

Manuscrit reçu le 3 septembre 2013 ; commentaires éditoriaux formulés à l'auteur le 11 novembre et le $1^{\text {er }}$ décembre 2013 ; accepté pour publication le 3 décembre 2013

\section{Mots-clés \\ Valeurs : \\ éthique ; \\ apprentissage ; \\ enseignement ; \\ transformationnel ; expérientiel ; \\ curriculum caché ; \\ professionnalisme}

\section{Keywords}

Values;

ethic;

learning;

teaching;

transformational;

experiential;

hidden curriculum;

professionalism
Messages clés. À différentes étapes de la formation d'un résident, l'impact du curriculum caché risque de l'éloigner des valeurs promues.

Pour influencer positivement le parcours du résident et l'aider à enrichir son savoir-être, le pédagogue devra créer un environnement favorable et sensibiliser le résident aux expériences qu'il vit en recourant à des stratégies pédagogiques diversifiées.

En semant le doute à l'égard de croyances inappropriées, il est possible d'induire une mise en mouvement vers des expériences nouvelles, intenses et diversifiées et de favoriser des apprentissages intégrant une dimension éthique.

Key messages. The impact of the hidden curriculum at various training stages may be steering residents away from encouraged values.

To positively influence residents' career, teachers need to develop a whole range of ways that help create a favorable environment and raise awareness to what residents will be experiencing.

By challenging rigid or inappropriate beliefs, it is possible to open residents to new, powerful and diversified experiences and accordingly promote a healthy integration of learning. 


\section{Introduction}

Le bon enseignant rend explicite son raisonnement clinique afin de servir de modèle aux étudiants en médecine et notamment, en contexte de stage postgradué, aux résidents. Devrait-il également rendre explicite l'importance des valeurs intimes qui influencent secrètement sa pratique ? Et devrait-il encourager le résident à découvrir et à dévoiler ses propres repères intérieurs, afin d'exercer à leur égard une certaine influence? Devrait-il inciter le résident à sortir de sa zone de confort avant que la vie ne l'oblige à le faire ?

Au moment de définir les compétences requises pour être un bon médecin de famille, le Collège des médecins prend position au sujet des caractéristiques recherchées chez ses membres. Ces caractéristiques présupposent l'acquisition de certaines valeurs. Nous adoptons ici la définition de Lieff ${ }^{[1]}$ qui décrit les valeurs comme étant les croyances au sujet de ce qui est bien et de ce que la conscience dicte comme étant les plus hautes priorités dans la vie. Les valeurs guident ou qualifient notre conduite personnelle, nos interactions avec les autres, l'implication et la satisfaction dans sa carrière.

Selon Borges et Hartung ${ }^{[2]}$, les valeurs promues au cours de la formation en médecine évoluent au cours du temps. L'altruisme, la responsabilité, l'honneur, l'intégrité, le sens du devoir et le respect d'autrui sont des vertus soutenues par le discours officiel de la médecine ${ }^{[3]}$. Les étudiants en médecine rapportent toutefois régulièrement des interactions ou des observations, au cours desquelles leurs enseignants ne respectent pas les règles hospitalières, violent la confidentialité du patient, formulent des commentaires dégradants au sujet des patients, manquent de respect envers des collègues médecins et ne fondent pas en priorité leurs décisions au regard du patient considéré comme finalité princeps de l'action médicale. Un manque de cohérence entre les messages formulés par les enseignants facultaires et ceux délivrés par les superviseurs dans les milieux cliniques peut nuire à l'intégration harmonieuse des valeurs. Face à une telle ambiguité, les étudiants peuvent être tentés de simplement faire semblant d'adhérer aux valeurs officielles, selon le contexte et les conséquences anticipées.
Coulehan et Williams ${ }^{[4]}$ affirment que le milieu de l'éducation médicale, considéré en tant qu'institution, prétend faire la promotion de l'empathie, de la compassion et de l'altruisme mais que dans les faits, ses acteurs font tacitement la promotion du détachement, de l'intérêt personnel et accordent peu de place à la prise en compte de la subjectivité. Dychel et Epstein $^{[5]}$, quant à eux, accusent l'éducation médicale d'éteindre la précieuse curiosité des étudiants.

Les propres valeurs du résident peuvent entrer en conflit. Être libre ou être au service du patient ? Être autonome ou satisfaire les attentes du supérieur hiérarchique ? Rester plus longtemps au travail ou rentrer à la maison ? La manière de prendre en compte les tensions entre les différentes valeurs, dans une recherche d'équilibre, évolue avec le temps. Selon Borges et Hartung $^{[2]}$, au fur et à mesure de leur formation, les résidents tendent à privilégier un mode de vie qui leur permet d'exercer un contrôle sur la gestion du temps et qui favorise un équilibre entre la vie familiale et professionnelle. Ils recherchent progressivement l'indépendance et l'autonomie.

\section{Créer un environnement favorable}

Lucey et Souba ${ }^{[6]}$ rapportent que le paradigme éducationnel traditionnel utilise des règles renforçant les valeurs désirées, sollicite des modèles de rôles positifs, récompense les comportements souhaités et élimine ceux qui transgressent les règles.

L'exposition des étudiants au milieu clinique leur offre en principe l'occasion de d'examiner comment les principes magistraux peuvent prendre leur sens et s'appliquer dans la vie réelle. Pourtant, les modèles de rôle rencontrés en milieu clinique ne sont pas toujours en harmonie avec ce qui est prôné en enseignement magistral. Au quotidien, l'influence de certains de modèles de rôle, par le processus d'imitation que ceux-ci induisent, peut s'exercer de façon opposée par rapport aux intentions de l'institution. Siristatidis et Gagari $^{[7]}$ affirment que leurs collègues enseignants en médecine n'ont pas la capacité d'enseigner en s'appuyant sur des données probantes récentes, qu'ils n'ont pas le temps de superviser les résidents, que 
sans gratification financière suffisante, ils ne sont pas motivés pour former les résidents, même s'ils réussissent à le faire croire de façon convaincante et, enfin, qu'ils ne sont pas intéressé à former qui que ce soit qui deviendrait par la suite plus compétent qu' eux. On peut se demander quelles valeurs transmettent ces modèles de rôle.

Mezirow ${ }^{[8]}$, quant à lui, souligne l'importance de la persuasion sociale pour modifier les valeurs des gens. Outre l'exemple que l'on peut donner, on peut convaincre en faisant avec persistance la promotion des valeurs désirées, par exemple au travers de compliments et de suggestions. Faire la morale ne suffit cependant pas. Le pédagogue souhaitera responsabiliser le résident en créant les conditions d'une confrontation des valeurs en jeu dans une situation avec les apprentissages antérieurs de l'étudiant, puis en enrichissant son univers intérieur par une réflexion éthique et déontologique. Les résidents seront davantage en mesure d'adopter un fonctionnement conforme aux règles du milieu s'ils connaissent les règles et les lois qui s'appliquent et les conséquences des transgressions ${ }^{[9]}$.

\section{Sensibiliser le résident}

On peut rendre explicites des valeurs à un étudiant par une rétroaction de qualité ${ }^{[9]}$. Des rétroactions convergentes de superviseurs, de pairs et de patients, relatives au manque d'empathie ou à l'indifférence d'un résident, auront une forte probabilité de le sensibiliser à sa réalité. Il est possible d'inviter un résident ambivalent à reconnaître les malaises et les dissonances associés à un conflit de valeurs ${ }^{[9]}$ et à percevoir l'écart entre son discours et son comportement. Il lui sera ainsi plus difficile de rester dans une posture de négation et de déni s'il reçoit de façon répétée une rétroaction congruente de la part de son environnement social et professionnel. Son évolution sera facilitée si l'on prend le temps de créer les conditions favorables à une pensée et une action réflexives ${ }^{[10-12]}$, c'est-à-dire en l'invitant à se projeter dans l'action et à se regarder agir, en prenant conscience de ce qu'il ressent et en observant l'impact de ses attitudes et de ses comportements.
Sa réflexion devra porter entre autres sur la recherche d'indicateurs tangibles au regard desquels il analysera s'il a atteint ses buts ${ }^{[13,14]}$. Quand sait-on que l'on est un bon résident ou un bon médecin ? Quand il n'y a plus d'anxiété face au patient ? Quand l'anxiété est identifiée et contrôlée ? Quand le patient nous remercie sincèrement? Quand le superviseur nous félicite? Quand le superviseur ne nous félicite plus mais nous traite plutôt d'égal à égal ? La réflexion sur ses critères personnels permet de nuancer ses réactions et d'en mieux comprendre la provenance. Certains auteurs suggèrent que c'est en devenant euxmêmes formateurs que les résidents découvriront la pleine nature de ce qu'ils savent et de ce qu'ils sont ${ }^{[16]}$.

\section{Semer le doute}

Constatant que certaines valeurs se modifient avec le temps, le résident reconnaîtra l'aspect relatif des valeurs qu'il a acquises au cours de son histoire de vie $^{[9]}$. Il y a avantage à prendre conscience que nos pensées et nos croyances ne sont que des hypothèses. Mezirow ${ }^{[8]}$ suggère de prendre le temps de vérifier ces hypothèses et d'en rechercher à la fois des preuves et des démentis. Y a-t-il d'autres façons de vivre ? D'autres choix ? Quels en sont les avantages et les inconvénients?

L'approbation sans réserve de ses professeurs peut aider un étudiant à « survivre » au début de ses études médicales mais le temps va lui apprendre qu'il est encadré par une variété de modèles et que l'approbation dépend du contexte, des écoles de pensées et du cheminement de chacun, ce qui le conduit à prendre un certain recul critique face aux choix présentés.

White ${ }^{[15]}$ souligne l'importance de déconstruire certaines valeurs problématiques avant de tenter d'en promouvoir une autre. De la même façon qu' on ne passe pas du sommet d'une montagne au sommet de la montagne suivante, il faudra parfois remettre en questions certaines croyances, descendre progressivement de son piédestal, marcher un certain temps vers la vallée, avant de reprendre la route vers le nouveau sommet qui nous attend. Le héros de la première année d'études en médecine ne sera pas nécessairement le 
héros de la fin de la formation. Les priorités changent. Les critères d'appréciation se modifient. La valeur d'obéissance aveugle laisse la place à un recul critique nuancé.

Legault ${ }^{[9]}$ suggère d'inviter un étudiant à explorer différents points de vue, grâce à des questionnements alternatifs, tels que : qu'est ce qui est souhaitable pour moi, pour autrui, pour la société, pour l'environnement ? Quel est le coût pour la société de fournir ce type d'examens onéreux ? Quelle conséquence aura l'obligation de consulter un spécialiste pour avoir accès à un examen qui pourrait être prescrit en première ligne ? Il conviendra alors d'examiner jusqu'à quel degré ces différents points de vue sont intériorisés et valorisés.

Nous souhaitons que le résident fasse évoluer son organisation mentale, émotionnelle et relationnelle d'une manière qui le prépare à affronter la complexité. Cela peut être favorisé en incitant l'étudiant à apprécier l'impact de ses croyances et de ses valeurs dans différents contextes, à différents moments, auprès de différentes personnes et dans différentes situations extrêmes ${ }^{[10]}$. Ainsi par exemple, nous nous sentons responsables de protéger des enfants de ce que nous considérons comme de la maltraitance. Mais sommes-nous responsables au point d'évaluer l'impact, pour eux et pour leur entourage, par exemple en contexte québécois, de la séparation des enfants des premières nations de leur héritage culturel ?

\section{Mettre en mouvement}

Le résident pourra se mettre plus facilement en mouvement si on le met au défi d'explorer des choix alternatifs et de s'exposer à des points de vue diversifiés ${ }^{[10]}$. On pourra lui fournir des occasions de partager des expériences nouvelles, qui suscitent le conflit cognitif ou affectif, et qui désorientent, et l'exposer à des expériences diversifiées et de grande intensité qui invitent chaque participant à la recherche de sens ${ }^{[10]}$. Il pourra ainsi prendre conscience que certaines de ses convictions ne sont que des préjugés, entretenus par des stéréotypes sociaux.

Les résidents de l'Université de Sherbrooke, au Québec, ont l'opportunité d'effectuer des stages en
Haïti ou au Mali. Confrontés à une culture où la mort d'un enfant n'est qu'un désagrément mais où la mort d'une personne âgée est une catastrophe, ils peuvent ainsi faire le constat de la relativité de leurs valeurs.

Il convient de préparer les résidents à la gestion de l'incertitude et de la complexité, et à la prise en compte des conflits de valeurs. Que répondront-ils, par exemple, lorsqu'ils seront confrontés à des situations de médecins qui donnent leur numéro de téléphone personnel à certains patients, qui ont fermé le dossier de leur ex-patiente pour développer avec elle une relation maritale qui dure depuis 15 ans ? Comment gèreront-ils l'absence de spécialistes auxquels soumettre les cas difficiles, ou la non disponibilité de plateaux techniques ou d'examens sophistiqués, grâce auxquels ils auraient pu poser leurs diagnostics avec davantage d'assurance?

Glee et Scouarnec ${ }^{[16]}$ rapportent qu'à partir d'un dilemme déstabilisant, l'individu traverse un processus de transformation qui l'amène à une réappropriation de sa propre vie. Les études recensées ont cependant montré qu'il était souhaitable de ne pas vivre de telles expériences dans un contexte d'isolement et que leur partage au sein d'un groupe constituait le moyen idéal pour pratiquer l'apprentissage par transformation. De telles conditions permettent aux apprenants de partager leurs histoires sociales, culturelles et politiques avec les autres, à l'intérieur d'une structure favorisant intentionnellement la collaboration et la démocratie ${ }^{[10]}$. La délibération en petit groupe favorise ainsi une réflexion en profondeur et une intégration à la fois plus explicite, plus nuancée et plus complexe des points de vue de toutes les personnes et institutions impliquées, ainsi que des lois et règles qui s'appliquent. Cela évite le raisonnement exclusivement émotionnel et impulsif.

Aronson ${ }^{[11]}$ suggère de préparer le développement de la pensée réflexive en structurant l'activité d'apprentissage de façon à ce que le but de l'activité soit explicite dans l'esprit du résident. À cette fin, l'élaboration d'un plan et l'anticipation d'un suivi de l'activité sont de nature à faciliter une meilleure intégration des apprentissages à partir de l'expérience. Les apprentissages transformationnels s'effectuent sur la durée et doivent être préparés. Il faudra notamment, au regard des préoccupations éthiques et professionnelles, 
documenter la solidité et l'ampleur des changements vécus et en vérifier l'impact au cours du temps ${ }^{[10]}$.

\section{Favoriser l'intégration}

La clarification de la hiérarchie des valeurs doit permettre de faciliter les choix ${ }^{[14,17]}$. Ainsi, un résident peut considérer que sa priorité est de prendre soin de son confort et de son état physique pour éviter l'épuisement, jusqu'à ce qu'un patient se présente en fin de journée dans un état de désorganisation. Il peut alors décider que sa priorité devient le patient, jusqu'à ce qu'un de ses propres enfants doive être conduit au service d'urgence à la suite d'une chute, ce qui le conduit à réviser sa décision et à considérer que sa priorité est désormais son fils, jusqu'à ce qu'il se rende compte que son rythme cardiaque est vraiment trop accéléré et qu'il doive consulter un collègue en urgence. En ayant explicitement à l'esprit qu'il convient d'identifier au nom de quoi décider, le résident peut prendre conscience de la variabilité de ses priorités et pourra élaborer des réponses à des questions telles que : «Qu'est-ce qui m'amènerait à renoncer à un choix pour en accepter un autre?»

Pour autant, il convient de sélectionner les situations d'enseignement et d'apprentissage avec discernement. Les conséquences d'interventions pédagogiques dans des contextes fortement mobilisateurs d'affects peuvent avoir l'effet contraire à celui que l'on désire. Ainsi, le contact du résident avec des toxicomanes peut favoriser le développement d'un intérêt ou exacerber une aversion et favoriser des stratégies d'évitement. L'intégration des valeurs sera favorisée par la préparation, l'encadrement et les échanges en groupe $^{[10,18]}$.

La réflexion sur les valeurs s'inscrit dans un projet éducationnel qui vise à obtenir par l'enseignement une transformation en profondeur de l'apprenant, à rebours d'une tendance à fragmenter la formation des médecins en un ensemble d'éléments mesurables. Jarvis-Selinger et al. ${ }^{[19]}$ encouragent une approche plus globale, se concentrant davantage sur l'identité, sur l' « être », plutôt que sur les habiletés, le « faire ».

\section{Conclusion}

Le bon enseignant en médecine, familier avec le concept de curriculum caché, ne laisse pas au hasard la transmission des valeurs propices à la pratique de la médecine. Il favorise la création d'un environnement favorable à l'apprentissage, incluant des lieux de partage et de délibération. Il sensibilise le résident à l'impact de ses valeurs et aux tensions suscitées par les conflits dans ce domaine. Il sème le doute face aux croyances erronées ou inappropriées, pour inviter le résident à mettre à jour, enrichir et complexifier sa compréhension de l'univers de la médecine. Il favorise l'évolution du résident par la présentation de défis adaptés, par l'exposition à des situations d'apprentissages nouvelles, authentiques et diversifiées. Le résident pourra ainsi travailler avec succès de manière féconde et apporter sa contribution à la communauté.

\section{Références}

1. Lieff SJ. Perspective: The Missing Link in Academic Career Planning and Development: Pursuit of Meaningful and Aligned Work, Acad Med 2003;84:1383-8.

2. Borges NJ, Hartung PJ. Stability of values during medical school. Med Teach 2010;32:779-81.

3. Hoiosko M, Skinner J, Mac Caughelty C, Morrissey Stahi K. Building the Implicit BSW Curriculum at a Large Southern State University. J Soc Work Educ 2010;46:411-23.

4. Coulehan J, Williams PC. Vanquishing Virtue: The Impact of Medical Education, Acad Med 2001;76:598-605.

5. Dychel L, Epstein R. Curiosity and medical education. Med Educ 2011;45:663-8.

6. Lucey CX, Souba W. The Problem With the Problem of Professionalism. Acad Med 2010;85:1018-24.

7. Siristatidis D, Gagari E. Residents' training and the uncomfortable truths: a program director's perpective. Archives of Gynecology \& Obstetrics 2011;284:785-6.

8. Mezirow J. How critical reflection triggers transformative learning. In: Mezirow, J. et al. (Eds.) Fostering Critical Reflection in Adulthood: A Guide to Transformative and Emancipatory Learning. San Francisco (CA): Jossey-Bass, 1990, 1-20. 
9. Legault GA. Professionnalisme et délibération éthique. Québec : Presses de l'Université du Québec, 2012.

10. Duchesne C. L'apprentissage par transformation en contexte de formation professionnelle. Éducation et francophonie 2010:38:33-50.

11. Aronson L. Twelve tips for teaching reflection at all levels of medical education. Med Teach 2010;33:200-205.

12. Duchesne C, Leurebourg R. La recherche-intervention en formation des adultes : une démarche favorisant l'apprentissage transformateur, Recherches qualitatives 2012;31:3-24.

13. Thiry A. Les trois types de coaching. Paris : De Boeck, 2007, 34

14. Vermersch P. L'entretien d'explicitation, Issy-lesMoulineaux : Éditeur ESF, 2011.

15. White M. Deconstruction and Therapy, In SG Gilligan \& R Price (Eds). Therapeutic conversations, New York: Norton, 1993, 22-61.
16. Glee C, Scouarnec A. Parcours professionnels et enjeux RH contemporains : Comment passer du subir au choisir? XIXe congrès de l'AGRH, Université de Dakar (UCAD), 2008, 9-25.

17. Andreas S, Andreas C. IV Shifting the Importance of Criteria. Change Your Mind And Keep the Change. Moab (UT): Real People Press, 1987, 61-87.

18. Chamberland G, Lavoie L, Marquis D. 20 formules pédagogiques. Québec : Presses de l'Université du Québec, 2009, 40.

19. Jarvis-Selinger S, Pratt DD, Reegehr G. Competency is Not enough: Integrating Identity Formation into the Medical Education Discourse. Acad Med 2012;87:1185-90.

Correspondance et offprints : Bruno Fortin. Unité de Médecine Familiale Charles-Lemoyne. 299 Bd. Sir-Wilfrid-Laurier, suite 201. J4R 2L1 Saint-Lambert (Québec), Canada.

Mailto : Bruno.Fortin@USherbrooke.ca 